\title{
Inégalités sociales et enjeux liés à la santé des aînés francophones en situation minoritaire : le cas de l'Atlantique et de l'Acadie des Maritimes
}

\author{
Majella Simard ${ }^{a}$, Louise Bouchard ${ }^{b}$
}

RÉSUMÉ. Le vieillissement démographique soulève des enjeux fondamentaux dans la réponse des acteurs sociaux aux besoins manifestés par les aînés sur le plan de la santé. L'objectif de cette contribution est double. Il consiste, d'une part, à identifier les principaux enjeux auxquels sont confrontés les aînés francophones en situation minoritaire sur le plan de la santé et, d'autre part, à examiner la situation des inégalités sociales et les conditions de vie qui en découlent comme déterminant de la santé, de la qualité et de l'accès aux services dans une perspective de développement territorial. Les résultats de notre analyse révèlent que le rapport minoritaire/majoritaire traduit une inégalité sociale qui, couplée à d'autres déterminants sociaux, contribue de facto à la formation de disparités sur le plan de la santé, d'où la nécessité de mettre en œuvre une politique à l'endroit des aînés vivant en milieu minoritaire francophone destinée à réduire de tels écarts.

\begin{abstract}
Demographic aging raises fundamental issues in the response of social actors to the health needs of seniors. The purpose of this contribution is twofold. It consists, on the one hand, of identifying the main health issues facing Francophone seniors in minority communities and, on the other hand, of examining the situation of social inequalities and the resulting living conditions as a determinant of health, as well as to the quality of and access to services from a territorial development perspective. The results of our analysis show that the minority/majority ratio reflects a social inequality which, coupled with other social determinants, contributes de facto to the formation of health disparities, hence the need to implement a policy for seniors living in Francophone minority communities designed to reduce such disparities.
\end{abstract}

\section{Introduction}

Le vieillissement de la population, défini comme l'augmentation de la proportion de personnes âgées de 65 ans ou plus au cours d'une période donnée, est un phénomène universel et irréversible. Bien qu'il se manifeste de manière différente selon les territoires, le vieillissement pose d'importants défis en matière de gouvernance territoriale, de logement, d'offre de services, de politiques et de finances publiques. Par exemple, en raison de l'augmentation de l'espérance de vie, l'accroissement du nombre de personnes âgées, une réalité mieux connue sous le vocable de "gérontocroissance ", est susceptible de faire surgir, chez les personnes âgées, de nouveaux besoins en matière de soins et de services, ce qui soulève des enjeux fondamentaux dans la réponse des divers acteurs sociaux en ce qui a trait à l'amélioration de la qualité de vie des aînés.

Même si le vieillissement ne représente pas, pour la majorité de la population, un obstacle au maintien d'une bonne santé en raison des progrès de la médecine et des conditions sociosanitaires, force est de reconnaitre que plusieurs aînés, plus nombreux en âge avancé, sont sujets à diverses vulnérabilités. Parmi celles-ci, mentionnons l'isolement, la solitude, la précarité financière, la présence de

\footnotetext{
${ }^{a}$ Professeur, Département d'histoire et de géographie, Université de Moncton

b Professeure, École d'études sociologiques et anthropologiques, Université d'Ottawa
} 
maladies chroniques concomitantes, etc. Ces vulnérabilités sont susceptibles de se manifester avec plus d'acuité en contexte linguistique minoritaire. À ce titre, différents travaux ont permis de mettre en exergue la persistance d'inégalités sociales et d'accès aux ressources au sein des communautés de langue officielle en situation minoritaire. Couplés à d'autres déterminants sociaux tels que le statut socio-économique, les niveaux d'éducation et de littératie en santé, l'âge, le sexe et l'immigration, ces inégalités entrainent indubitablement des disparités spatiales, ce qui s'inscrit en porte-à-faux avec un développement territorial durable.

L'objectif de cette contribution est double. Il consiste, en premier lieu, à identifier les principaux enjeux auxquels font face les aînés francophones (en particulier ceux de l'Atlantique et de l'Acadie des Maritimes) en situation minoritaire sur le plan de la santé en croisant certaines variables issues d'analyses secondaires de l'Enquête sur la santé dans les collectivités canadiennes (ESCC - cycles 2003 à 2012), de l'Étude canadienne de suivi de la mortalité (1991 à 2006) et des Recensements canadiens (1981 et 2016). Dans un second temps, il vise à examiner la situation des inégalités sociales ainsi que les conditions de vie qui en découlent comme déterminant potentiel de la santé, de la qualité et de l'accès aux services dans une perspective de développement territorial.

Notre contribution se décline en cinq parties. Dans un premier temps, nous exposons les principaux concepts qui jalonneront notre analyse. Par la suite, nous dépeignons brièvement l'état de la langue et l'évolution du vieillissement au Canada, en Atlantique et en Acadie. En troisième lieu, nous esquissons la problématique des inégalités sociales en santé au Canada. Ces dernières ainsi que les enjeux liés à la santé font l'objet de la quatrième partie. Notre regard se tournera plus spécifiquement autour de l'éducation, du revenu, de la mortalité et de la pauvreté, ainsi que des paramètres liés à la langue, au genre et au niveau de littératie en santé des aînés francophones en situation linguistique minoritaire. Enfin, dans la cinquième partie, nous examinerons la problématique des inégalités sociales en Atlantique et en Acadie.

\section{Concepts}

Le développement est compris comme un processus de changement à la fois social, économique, voire culturel prenant assise le plus souvent - mais non exclusivement - en amont, c'est-à-dire de la base, en vue d'améliorer la qualité de vie des individus qui résident au sein du territoire (El-Batal et Joyal, 2015; Klein et Champagne, 2011; Tremblay, Klein et Fontan, 2009). Le changement ainsi produit doit s'inscrire dans une perspective d'amélioration du cadre (environnement naturel, équipements publics), du milieu (vie sociale et culturelle), du niveau de vie (revenu, pouvoir d'achat) et, donc, des conditions de vie des individus qui occupent ce territoire (Simard, 2018, 2003).

Par conséquent, le développement territorial est un processus qui implique une utilisation optimale des ressources, une réduction des inégalités, une plus grande justice sociospatiale et une meilleure redistribution de la richesse en vue de favoriser l'épanouissement intégral des individus et des collectivités (Blanchet, 2014; Simard, 2003, 1999). L'épithète «durable » associée à la notion de développement territorial implique non seulement une certaine continuité dans le déroulement du processus, mais aussi une harmonisation entre les composantes sociale, environnementale et économique du développement (Commission mondiale sur l'environnement et le développement, 1988).

Étant donné que le développement économique s'effectue suivant une logique néolibérale, des inégalités subsistent entre les individus et les territoires (Proulx, 2019). En effet, tous les individus ne disposent pas des mêmes ressources, des mêmes chances ou des mêmes talents. De plus, les ressources naturelles, l'organisation spatiale et les politiques publiques diffèrent considérablement d'un milieu à l'autre (Proulx, 2019; Meloche, 2012; Dugas, 1996). Il en est de même des facteurs intangibles liés au développement tels que la résilience, l'esprit identitaire, le leadership ou le capital social, qui se manifestent différemment selon les endroits (Simard, 2018; Epanda, 2003). De façon générale, nous observons une corrélation entre le développement économique et le bien-être social. Par exemple, l'état de santé des individus tend à s'amenuiser en fonction de la réduction de leur niveau de revenu (Moleux, Schaetzel et Scotton, 2011; OMS, 2018a, 2018b). En outre, l'écart entre les riches et les pauvres permet d'expliquer les différences observées en ce qui a trait à l'espérance de vie, et ce, à différents échelons géographiques. À cet égard, l'échelle des inégalités peut également servir 
d'explication concernant l'état de bien-être physique et psychologique des individus (Wilkinson et Pickett, 2013). Le creusement des inégalités a aussi des impacts sociaux non négligeables, que ce soit sur le plan de l'éducation ou de la culture. Ainsi, des écarts trop prononcés sont susceptibles d'alimenter les frustrations, le cynisme, le populisme, voire la xénophobie (Zorn, 2017).

Dans un système basé sur l'économie de marché, un minimum d'inégalités est inévitable. Selon John Rawls (2006), une société peut s'accommoder plutôt bien des inégalités économiques, à la condition toutefois que l'égalité des chances soit assurée et que ces écarts ne contribuent pas à amenuiser la qualité de vie des individus plus démunis de la société. Autrement dit, les inégalités sont acceptables dans la mesure où elles contribuent à la maximisation des individus les plus désavantagés. C'est ce que Rawls appelle le principe du maximin.

Prôné par l'Organisation mondiale de la santé (OMS), le vieillissement en santé est "défini comme le processus de développement et de maintien des capacités fonctionnelles qui permettent aux personnes âgées de jouir d'un état de bienêtre »(OMS, 2015, p. 14). Le vieillissement en santé a toutes sortes d'impacts, tant sur les plans social, économique, territorial qu'environnemental. Sur le plan social, par exemple, la participation et l'intégration des personnes âgées à la vie communautaire, en particulier à travers le bénévolat, favorisent un vieillissement en santé (Raymond, Gagné, Sévigny et Tourigny, 2011). Sur le plan économique, celui-ci est également susceptible de réduire les coûts liés au système de santé (Saillant, 2016). Travailler plus longtemps peut également être bénéfique sur la santé physique et psychologique des aînés (Cardinal, Langlois, Gagné et Touriginy, 2008; Lagacé, 2007). Sur les plans territorial et environnemental, la qualité du cadre bâti ainsi que la proximité et l'accessibilité par rapport aux biens et aux services, telles qu'elles sont promues par le programme Municipalités amies des aînés, contribuent aussi à optimiser les possibilités de vieillir en bonne santé (Lord et Piché, 2018; Audet, Paris, Garon et Dumas, 2017).

Enfin, d'un point de vue sociologique, le concept de minorité, voire de groupe en situation minoritaire est appréhendé dans ses dimensions qualitatives, si l'on considère la dynamique du rapport minoritaire/majoritaire comme conscience collective, tensions, résistances, statut, marginalisation, dévalorisation, infériorité, domination et accès inégal au pouvoir. La notion renvoie aussi à l'existence d'« avantages » ou d'« opportunités » dont bénéficieraient certains milieux (Blanchet, 2005). Ainsi, le libellé « communautés de langue officielle en situation minoritaire», adopté par le gouvernement canadien, traduit bien la coexistence "problématique » et l'affrontement inégal qui persistent entre les deux langues officielles au Canada. Les caractéristiques géographiques du pays (dispersion de la population, faiblesse de l'armature urbaine dans l'Est du Canada, prédominance du fait rural par rapport au monde urbain, etc.) contribuent aussi à amplifier les impacts liés à la persistance de disparités, notamment en ce qui a trait à l'offre de services (Simard, 2015).

\section{2. État de la langue française et du vieillissement au Canada, en Atlantique et en Acadie des Maritimes}

Au dernier recensement de 2016, 947040 Canadiens déclaraient le français comme langue maternelle et vivant à l'extérieur du Québec (Statistique Canada, 2016b). Ces derniers se distribuaient comme suit: 490720 francophones en Ontario (3,7\% de la population), 231110 au NouveauBrunswick (31,4\%), 72155 en Alberta (1,8\%), 57425 en Colombie-Britannique (1,2\%), 40525 au Manitoba (3,2\%), 29465 en Nouvelle-Écosse (3,2\%), 15095 en Saskatchewan $(1,4 \%)$ et 4865 à l'Île-du-Prince-Édouard (3,4\%). Pour leur part, Terre-Neuve-et-Labrador $(0,5 \%)$, le Yukon (4,4\%), les Territoires du Nord-Ouest $(2,8 \%)$ et le Nunavut $(1,7 \%)$ comptaient 5690 francophones. À l'échelle des provinces maritimes ${ }^{1}$, le français constituait la langue maternelle d'au moins $20 \%$ de la population au sein de 105 localités, celles-ci correspondant, pour l'essentiel, au territoire de l'Acadie (voir figure 1), territoire qui correspondra, dans le cadre de cette contribution, à l'« Acadie des Maritimes ».

Comme ailleurs en Occident, le Canada est affecté par le vieillissement de sa population. La proportion des personnes âgées de 65 ans ou plus s'est effectivement accrue de 4,8 points de pourcentage entre 1981 et 2016 (voir figure 2). Les provinces de l'Atlantique, en particulier Terre-Neuve-et- 
Labrador, sont particulièrement concernées par ce phénomène (Simard, 2020). Or, les recensements de 1981 et de 2016 de Statistique Canada révèlent que c'est surtout en Acadie des Maritimes où le vieillissement se manifeste avec le plus d'acuité, la proportion des personnes âgées de 65 ans ou plus ayant augmenté de 14 points de pourcentage au cours de cette même période. Cette constatation nous amène donc à établir une corrélation entre le vieillissement et la langue, la population francophone étant plus affectée par ce phénomène.

En distinguant les milieux urbains et ruraux, nous constatons, à la figure 3 , que ceux-ci (14,2 points) sont plus touchés par le vieillissement que ceux-là (13,6 points). Les petites localités, c'est-à-dire celles dont la population est inférieure à 500 habitants, ont même affiché une augmentation de 16 points de pourcentage de leur proportion d'aînés entre 1981 et 2016. Au surplus, cette évolution a été supérieure à 20 points de pourcentage dans 10 localités acadiennes identifiées par leur toponyme à la figure 4. Parmi ces dernières, sept correspondent à des milieux ruraux. Leur taille démographique moyenne s'établit à 996 habitants. Leur taux d'activité $(53,7 \%)$ est inférieur à la moyenne de l'Atlantique (55,6 \%). Il en est de même en ce qui a trait au revenu perçu sous la forme de transferts gouvernementaux, qui atteint $28,7 \%$, comparativement à 23,7\% pour l'Atlantique. Enfin, le revenu moyen des ménages ne correspond qu'à $93,5 \%$ de celui des provinces de l'Atlantique. Il s'ensuit que la population francophone vivant en contexte minoritaire en Acadie des Maritimes fait face non seulement à un vieillissement plus prononcé, mais aussi à la persistance de nombreuses disparités sur le plan socio-économique. Dès lors, «vieillir en bonne santé » est susceptible de représenter un enjeu de taille, particulièrement pour les personnes âgées résidant en milieu rural acadien.

\section{Inégalités sociales en santé au Canada : minorisation linguistique et disparité de revenus}

Bien que le gouvernement du Canada reconnaisse l'existence de deux langues officielles titulaires de droits égaux, l'« égalité formelle» de celles-ci ne se traduit pas toujours par une "égalité réelle» (Landry, 2014, p. 2). Même s'il est doté d'un système de santé universel, le Canada est affecté par de nombreuses disparités sur le plan de la santé. Avec un revenu total des ménages de 100300 \$ en 2015 , qui correspond à $123,9 \%$ de celui du pays, l'Alberta figurait parmi la deuxième province la plus riche au dernier recensement de 2016. Cette même province arrivait au cinquième rang au Canada quant à l'espérance de vie à la naissance de ses résidents au cours de la période 2015-2017, et ce, autant chez les hommes (79,3 ans) que chez les femmes (83,8 ans) (Statistique Canada, 2020; 2016a).

À l'inverse, pour cette même période, l'espérance de vie à la naissance était plus faible au NouveauBrunswick, où les hommes pouvaient espérer vivre 78,6 ans et les femmes, 82,9 ans (Statistique Canada, 2020; 2016a). La situation apparaît encore plus précaire au Nunavut, où, pour la même période, l'espérance de vie était de 70,8 ans pour les hommes et de 73,4 ans pour les femmes (Statistique Canada, 2020; 2016a). En concomitance à ce fait, le Nunavut détenait, en 2015, le revenu total le plus faible (67 $860 \$$ ), ce qui n'équivaut qu'à 83,8\% de celui du Canada. Or, une forte proportion de la population de ce territoire se réclame une identité autochtone, surtout inuite. 


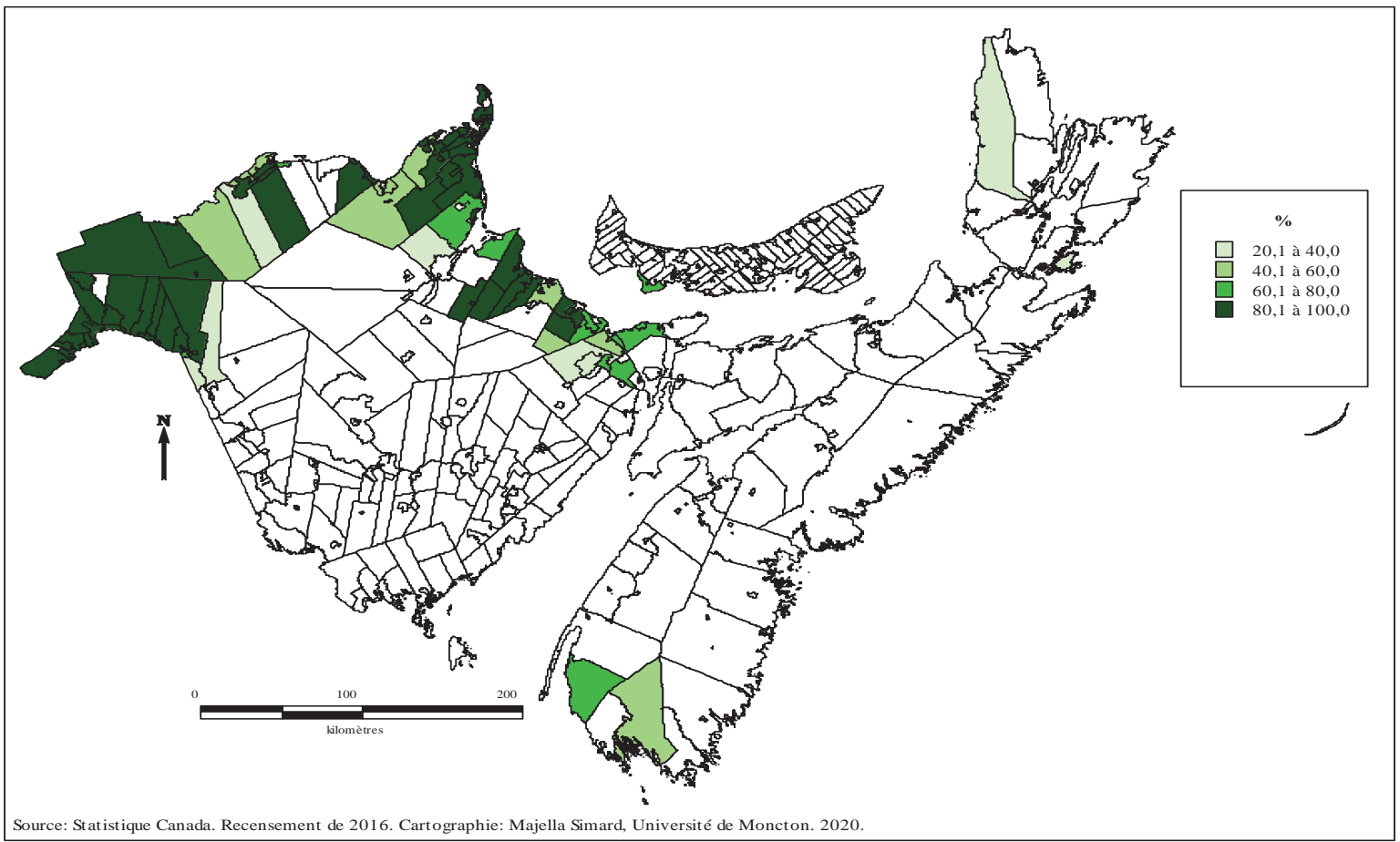

Figure 1 - Localité de l'Acadie des Maritimes dont plus de $20 \%$ de la population ont déclaré le français comme langue maternelle en 2016

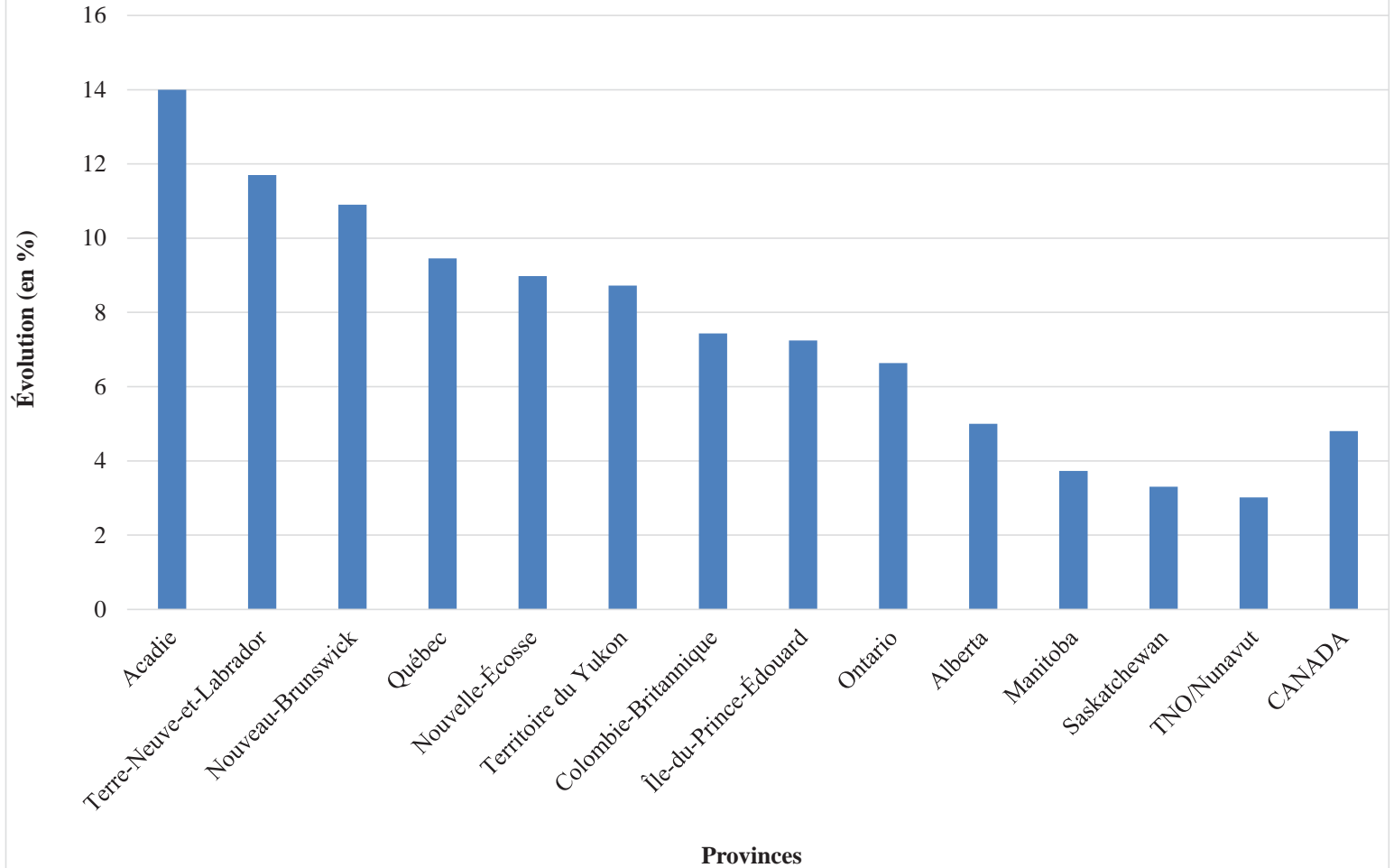

Source: Statistique Canada. Recensements de 1981 et de 2016. Compilation et calculs: Majella Simard, 2020.

Figure 2 - Évolution de la proportion des personnes âgées de 65 ans ou plus entre 1981 et 2016 dans les provinces canadiennes 


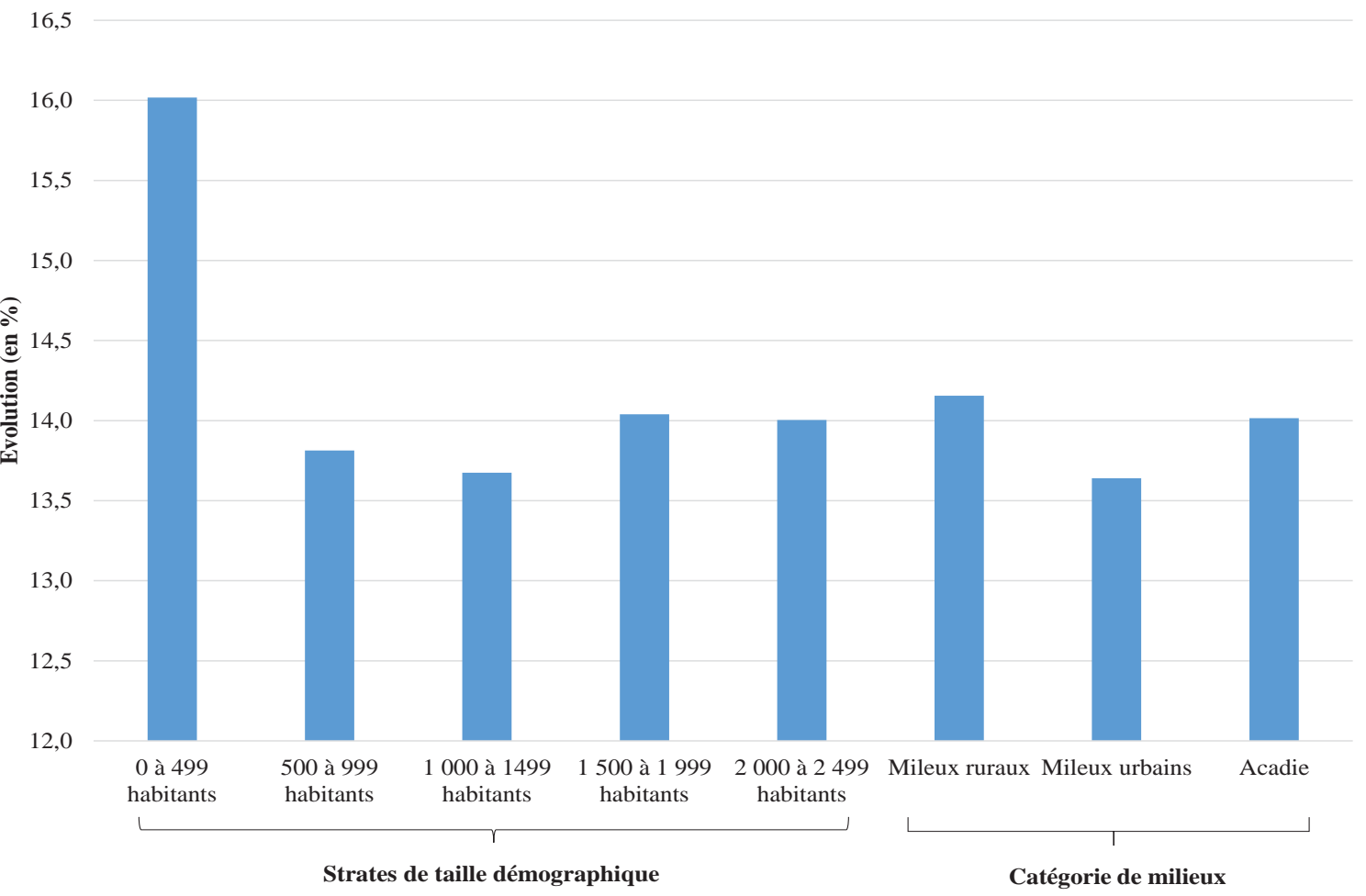

Source: Statistique Canada. Recensements de 1981 et de 2016. Compilation et calculs: Majella Simard, 2020.

Figure 3 - Évolution de la proportion des personnes âgées de 65 ans ou plus entre 1981 et 2016 en Acadie des Maritimes par strate de taille démographique et par catégorie de milieux

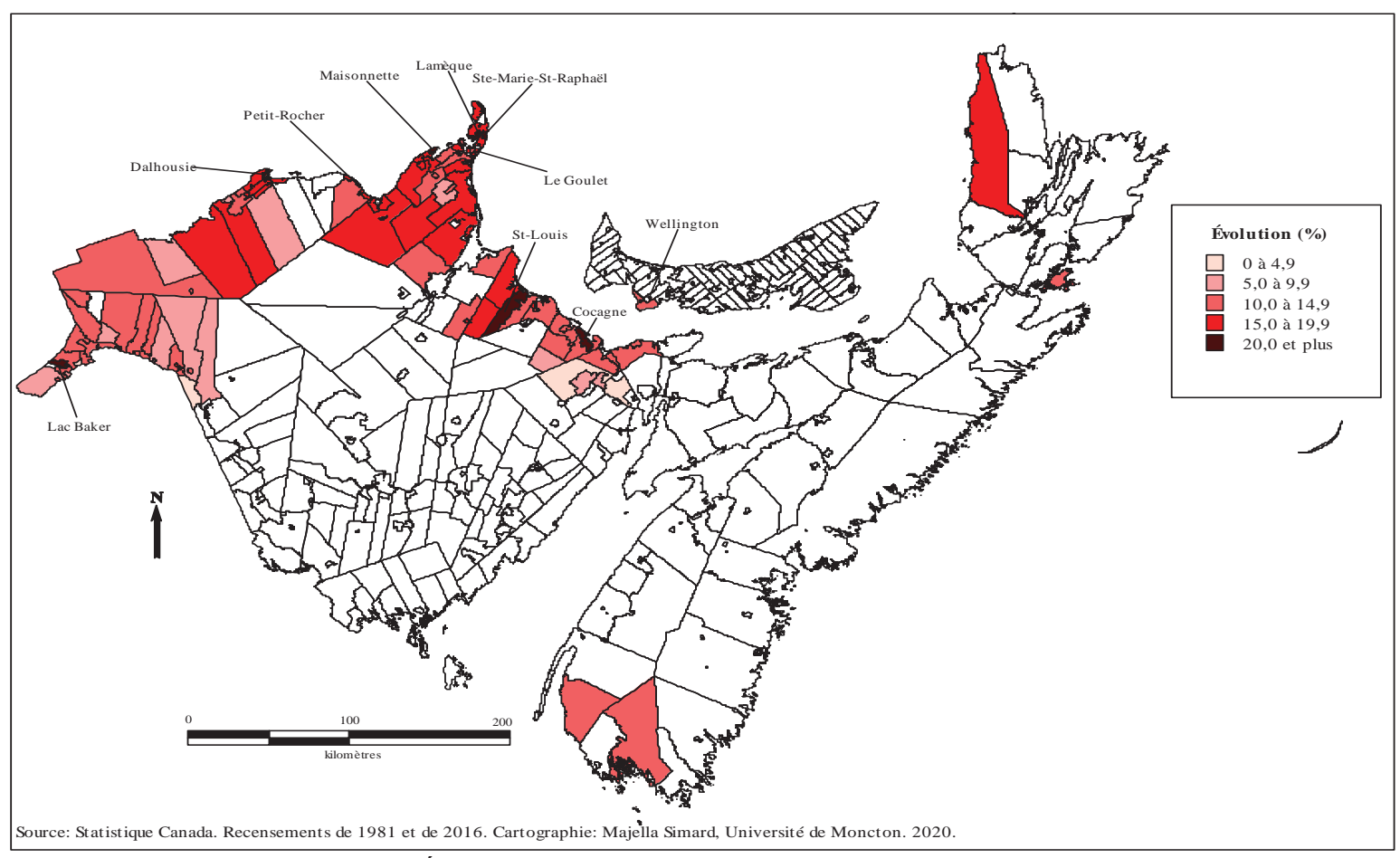

Figure 4 - Évolution de la proportion de personnes âgées de 65 ans ou plus au sein des localités de l'Acadie des Maritimes entre 1981 et 2016 
Dans leurs travaux, Wilkins, Tjepkema, Mustard et Choinnière (2008) ont montré l'existence d'un gradient significatif selon le niveau de revenu et l'espérance de vie. Ainsi, cette dernière augmente à chaque quintile de revenu successivement plus riche. La différence d'espérance de vie entre les quintiles de revenu le plus riche et le plus pauvre était de 6,8 ans chez les hommes et de 4,3 chez les femmes. De leur côté, Tjepkema, Wilkins et Long (2012) se sont attardés à la magnitude du gradient selon le niveau de scolarité, qui indique qu'il est plus élevé pour les individus dont le degré scolarisation est le plus faible. En clair, les auteurs constatent une surmortalité de $27 \%$ chez les hommes et de $22 \%$ chez les femmes faiblement scolarisés (Tjepkema et collab., 2012).

\section{Inégalités sociales et enjeux liés à la santé des aînés francophones en situation minoritaire}

La littérature portant sur la santé des populations a illustré à partir de moult exemples que l'âge, le sexe, le revenu, le niveau d'éducation ainsi que la position sociale de l'individu et du groupe auquel il appartient constituent les principaux déterminants de la santé, auxquels s'ajoute le fait de vivre en situation linguistique minoritaire (Bouchard, Gaboury, Chomienne, Gilbert et Dubois, 2009). Ainsi, les inégalités de santé résulteraient principalement de la répartition inégale des positions sociales, du statut qu'on leur accorde ainsi que de forces structurelles en amont, telles que la nature de l'économie et la gouvernance en particulier pour ce qui concerne les politiques publiques (OMS, 2018a). Il s'ensuit l'existence de relations causales entre chacun de ces indicateurs, lesquelles sont susceptibles de se traduire par des inégalités sur le plan de la santé. Dès lors, réduire de tels écarts devient un impératif incontournable dans la recherche d'une plus grande justice sociale et d'un développement territorial plus respectueux de la personne humaine (Simard, 2015; OMS, 2018a, 2018b).

À défaut de pouvoir analyser la totalité des variables qui influent sur les inégalités sociales de santé en contexte minoritaire francophone, nous traiterons plus spécifiquement de la relation entre l'éducation, le revenu, la mortalité et la pauvreté ainsi que des paramètres liés à la langue, au genre et au niveau de littératie en santé.

\section{1 Éducation, revenu, mortalité et pauvreté}

En premier lieu, le tableau 1 illustre la présence de disparités au chapitre de l'éducation et ce, à différents niveaux. Ainsi, comparativement au Canada, les résidents des provinces maritimes apparaissent globalement moins scolarisés, ces derniers étant proportionnellement plus nombreux à ne disposer d'aucun diplôme. Nous observons aussi l'existence d'inégalités en ce qui concerne la formation universitaire. Cette faiblesse, sur le plan éducationnel, affecte de manière particulière les personnes âgées et, plus spécifiquement, celles du Nouveau-Brunswick, cette dernière province apparaissant la moins scolarisée.

Par ailleurs, force est de constater qu'en situation de langue officielle minoritaire, la population francophone est plus âgée, vit surtout en milieu rural, possède une plus faible éducation, est plus susceptible de travailler dans un métier non spécialisé et, en conséquence, de détenir un plus faible niveau de revenu. Ainsi, selon Wilkins et ses collaborateurs (2008), les hommes dont la langue maternelle est le français avaient un taux de mortalité légèrement supérieur $(+13 \%)$ à celui de leurs homologues dont la langue maternelle est l'anglais. Par ailleurs, le taux de mortalité des personnes bilingues était le même que celui du groupe de référence. Des travaux menés sur la population francophone de langue officielle en situation minoritaire utilisant les données issues de l'Étude canadienne de suivi de la mortalité 1991-2006 arrivent à des résultats similaires (Sucha, Silva, Batista et Bouchard, 2015). Ainsi, la mortalité est plus élevée chez les femmes francophones n'ayant pas de diplôme d'études secondaires $(+35 \%$ par rapport au groupe de référence ayant un diplôme universitaire), alors que, chez les femmes anglophones, elle serait de $28 \%$ supérieure. Qui plus est, une corrélation se dessinerait entre la langue, la scolarité et le niveau de revenu. Dès lors, la mortalité serait plus élevée de $46 \%$ chez les hommes francophones à faible revenu par rapport à la catégorie des plus riches. Pour ce qui est de leurs homologues anglophones, elle serait de $42 \%$ plus élevée. Le fait d'être sans emploi occasionnerait une surmortalité de $75 \%$ chez les hommes francophones, de $60 \%$ chez les hommes anglophones, de $60 \%$ chez les femmes francophones et de $40 \%$ chez les femmes anglophones. La différence d'espérance de vie restante à 25 ans entre les quintiles de revenu le plus riche et le plus pauvre est de 7,4 ans chez les hommes francophones et de 3,8 ans chez les femmes (Sucha et collab., 2015). 


\begin{tabular}{|l|r|r|}
\hline \multicolumn{3}{|c|}{ Aucun certificat diplôme ou grade } \\
\hline & 15 ans et plus & 65 ans et plus \\
\hline Île-du-Prince-Édouard & 19,1 & 28,7 \\
\hline Nouveau-Brunswick & 22,0 & 34,7 \\
\hline Nouvelle-Écosse & 19,8 & 29,5 \\
\hline CANADA & 18,3 & 27,3 \\
\hline \multicolumn{2}{|c|}{ Certificat, diplôme ou grade universitaire au niveau du baccalauréat ou supérieur } \\
\hline \multicolumn{2}{|r|}{15 ans et plus } & 65 ans et plus \\
\hline Île-du-Prince-Édouard & 15,0 & 12,9 \\
\hline Nouveau-Brunswick & 13,0 & 11,1 \\
\hline Nouvelle-Écosse & 16,3 & 13,7 \\
\hline CANADA & 18,9 & 14,9 \\
\hline
\end{tabular}

Tableau 1 - Niveaux de scolarité (en \%) des personnes âgées de 65 ans ou plus en comparaison avec celles de 15 ans ou plus au sein des provinces maritimes et du Canada en 2016

Source : Statistique Canada, Recensement de la population de 2016, produit numéro 98-400-X2016242 au catalogue de Statistique Canada. Calculs de l'auteur. Repéré à https://www12.statcan.gc.ca/census-recensement/2016/dp-pd/dt-td/Rp-

fra.cfm?TABID =2\&LANG=F\&APATH=3\&DETAIL=0\&DIM=0\&FL=A\&FREE $=0 \& G C=0 \& G K=0 \& G R P=1 \& P I D=110634 \& P R I D=10$ $\& \mathrm{PTYPE}=109445 \& \mathrm{~S}=0 \& \mathrm{SHOWALL}=0 \& \mathrm{SUB}=0 \& \mathrm{Temporal}=2017 \& \mathrm{THEME}=123 \& \mathrm{VID}=0 \& \mathrm{VNAMEE}=\& \mathrm{VNAMEF}=$

En plus d'un vieillissement accéléré, les personnes âgées francophones minoritaires sont assujetties à une précarisation économique plus accentuée, comparativement à leurs homologues de la majorité anglophone. Ainsi, la langue et l'appartenance à la minorité francophone constituent des déterminants de la pauvreté chez les aînés (Bouchard et collab., 2015). Selon les analyses secondaires de l'ESCC (période de 2003 à 2012) que nous avons effectuées ${ }^{2}, 34 \%$ des francophones âgés de 65 ans et plus qui résident en Atlantique se situaient dans le quintile de revenu le plus faible, contre $30 \%$ des anglophones de cette même région et $28 \%$ pour l'ensemble des personnes âgées du Canada.

Au surplus, comme nous l'avons vu, les populations francophones sont, en contexte minoritaire, généralement moins scolarisées et plus sujettes au vieillissement. En effet, $44 \%$ des francophones de l'Atlantique âgés de 65 ans ou plus ne possédaient pas de diplôme d'études secondaires, comparativement à $29 \%$ chez les anglophones et à $23 \%$ pour l'ensemble des Canadiens appartenant au même groupe d'âge. En Acadie des Maritimes, cette proportion atteint 32,9\% dans les 10 localités les plus affectées par le vieillissement. Dans bien des cas, ces francophones résident au sein de milieux ruraux éloignés des villes, plus affectés par le vieillissement démographique et dont l'économie présente des signes de fragilité, autant de facteurs qui contribuent à entraver leur accès aux différents services disponibles, en particulier à ceux relevant de la santé (Simard et collab., 2015; Bouchard et Desmeules, 2011; Bouchard et Leis, 2008).

\subsection{Langue et genre}

En ce qui concerne plus spécifiquement les incidences de la langue, des travaux portant sur les barrières linguistiques et culturelles en santé, notamment chez les communautés de langue officielle en situation minoritaire, ont mis en exergue l'intérêt de nombreux chercheurs en sciences sociales sur les déterminants sociaux (Bouchard et Desmeules, 2011; Bowen, 2001). En outre, ces mêmes recherches ont révélé que les barrières linguistiques se répercuteraient négativement sur l'accessibilité et l'utilisation des services (Flores, 2006; Yeo, 2004; Sarver et Baker, 2000), sur l'état de santé des populations (Leis et Bouchard, 2013; Bouchard et collab., 2009) ainsi que sur la qualité des soins et des services offerts, voire sur la sécurité des patients (Bowen, 2015; Woloshin, Schwartz, Katz et Welch, 1997).

Au surplus, la minorité francophone aurait tendance à se percevoir en moins bonne santé, comparativement à la majorité anglophone, et ce, tant chez les hommes que chez les femmes (Bouchard et collab., 2009). Toutefois, en couplant cette corrélation aux principaux déterminants de la santé, il demeure, chez les hommes francophones, un « résiduel » de disparité, que nous pourrions attribuer au facteur «vie en situation minoritaire ». Par ailleurs, Bélanger et ses collaborateurs (2011), qui ont répliqué à l'échelle du Nouveau-Brunswick l'étude de Bouchard et ses collègues (2009), n'ont pas pu établir cette corrélation, bien que les hommes et les 
femmes francophones soient moins enclins que les anglophones à rapporter une bonne santé.

D'autres travaux attestent de la persistance d'inégalités socio-économiques en matière de santé plus marquées chez les hommes que chez les femmes pour des indicateurs tels que la mortalité, la morbidité et la santé perçue (Hunt et Macintyre, 2000; Wilkins et collab., 2008; Bouchard et collab., 2009). À l'inverse, proportionnellement plus nombreuses $(56 \%)$ que les hommes $(44 \%)$ à vivre en situation minoritaire, les femmes seraient plus susceptibles de souffrir d'isolement; $38 \%$ de celles-ci vivent seules, contre $19 \%$ des hommes. Elles seraient aussi proportionnellement plus nombreuses à ne pas détenir de diplôme d'études secondaires (37\%, comparativement à $19 \%$ des hommes) et à se situer dans le quintile de faible revenu (36\%, contre $24 \%$ des hommes) (Van Kemenade, Bouchard et Bergeron, 2015).

\subsection{Niveau de littératie en santé}

D'entrée de jeu, rappelons que la littératie en santé renvoie, selon Kickbush, Pelikan, Apfel et Tsouros (2013), à la connaissance générale en matière de santé, à la compréhension de ses enjeux et à la capacité des individus à obtenir les services nécessaires au maintien de leur santé et à appliquer correctement des directives médicales et/ou pharmaceutiques. Or, force est d'admettre que les personnes les plus susceptibles de présenter un niveau lacunaire de littératie en santé apparaissent également moins scolarisées. En Atlantique, toujours selon les données de l'ESCC, $44 \%$ des francophones âgés de 65 ans ou plus n'ont pas terminé leurs études secondaires. Cette proportion s'établit à $34 \%$ chez les francophones âgés vivant en situation minoritaire et à $23 \%$ chez l'ensemble des Canadiens âgés de 65 ans ou plus (Bouchard et Desmeules, 2017).

Pour les personnes âgées, la littératie en santé exerce un rôle fondamental au vieillissement en santé. Cet enjeu prend une signification particulière en situation linguistique minoritaire (Bouchard et collab., 2012). Qui plus est, au sein de ces milieux, il semble persister des difficultés d'accès à des services sociaux et de santé linguistiquement et culturellement adaptés, particulièrement en région rurale. De telles carences entraînent des difficultés accrues de communication avec les professionnels de la santé, ce qui aggrave du coup les effets néfastes des barrières linguistiques sur la santé. Le faible niveau de littératie en santé des personnes âgées a également de fortes incidences sur leur état de santé, une situation qui semble caractériser plus spécifiquement les milieux en contexte linguistique minoritaire.

Le modèle conceptuel qu'a produit le Bureau régional européen de l'OMS décline les sous-dimensions de la littératie en santé, lesquelles touchent l'accessibilité et la capacité d'obtenir de l'information, de la comprendre, de l'évaluer et de l'appliquer, notamment en ce qui a trait aux soins et aux services, à la prévention de la maladie et à la promotion de la santé (Kickbusch, Pelikan, Apfel et Tsouros, 2013). Par ailleurs, force est de reconnaittre qu'une littératie lacunaire en matière de santé (ou une incompréhension partielle relative à ses enjeux et à son maintien) a à la fois des effets directs (incapacité de lire ou de comprendre une ordonnance médicale) et indirects (stress, vulnérabilité, mauvaises habitudes de vie, périodes d'hospitalisation plus longues et fréquentes) sur la qualité de vie des personnes âgées (Racine, 2008).

\section{Inégalités sociales en santé en Atlantique et en Acadie des Maritimes}

Eu égard aux déterminants sociaux de la santé, la région de l'Atlantique, toujours selon les données de l'ESCC, comporte un plus fort contingent de personnes pauvres et compte davantage d'individus moins scolarisés. Quant au profil santé qui se dégage des analyses secondaires de cette même enquête, quelques indicateurs distinguent les aînés francophones des anglophones au sein des provinces maritimes. Par exemple, en ce qui concerne les représentations associées à la santé, les aînés francophones étaient proportionnellement plus nombreux à se percevoir en mauvaise santé $(31 \%$ contre $29 \%$ des anglophones et $25 \%$ des aînés canadiens) et en mauvaise santé mentale $(8 \%$ contre $5 \%$ des anglophones et $6 \%$ des Canadiens). Ils seraient aussi plus touchés par l'anxiété ( $6 \%$ contre $4 \%$ des anglophones et $4 \%$ des Canadiens). Les aînés francophones ont déclaré en plus grand nombre avoir eu besoin de soins de routine $(74 \%$ contre $69 \%$ des anglophones et $64 \%$ des aînés canadiens). Les aînés francophones des provinces maritimes seraient un peu plus nombreux à recevoir des soins à domicile $(20 \%$ contre $17 \%$ des anglophones). 
Par contre, pour d'autres indicateurs autodéclarés de la santé, les aînés francophones sembleraient mieux se porter ou sont comparables aux anglophones de l'Atlantique. C'est le cas notamment pour les variables suivantes: besoin d'aide pour effectuer ses tâches quotidiennes ( $29 \%$ contre $28 \%$ ); en surpoids et obèses $(57 \%$ contre $62 \%)$; inactifs $(60 \%$ contre $65 \%$ ); fumeurs réguliers ou occasionnels ( $8 \%$ contre $12 \%)$; buveurs quotidiens $(10 \%$ contre $14 \%)$; hypertension artérielle (49\% contre $52 \%$ ); diabète (16\% contre $21 \%)$; maladie cardiaque $(17 \%$ contre $22 \%$ ); besoin de soins spécialisés $(33 \%$ contre $39 \%$ ); et besoin de soins immédiats ( $22 \%$ contre $21 \%$ ).

Afin d'examiner plus attentivement si l'inégalité sociale se traduit par des écarts en matière de santé au sein des provinces de l'Atlantique, il serait opportun d'examiner de plus près la situation des aînés francophones moins éduqués et à faible revenu. Or, les faibles échantillons de francophones ne nous ont pas permis de conduire des analyses aussi fines de l'impact des déterminants sociaux sur la santé.

Bien que le Nouveau-Brunswick soit une province officiellement bilingue, une étude effectuée par Gagnon-Arpin, Bouchard, Leis et Bélanger (2014) révélait que $45 \%$ des francophones résidant au centre et au sud-ouest de la province considéraient comme difficile, voire impossible d'obtenir des services en français, contre $11 \%$ pour l'ensemble des francophones. À l'île-du-Prince-Édouard et en NouvelleÉcosse, ces proportions étaient respectivement de $66 \%$ et de $57 \%$ (Gagnon-Arpin et collab., 2014). De plus, non seulement les communautés francophones en situation minoritaire seraient plus vieillissantes et moins bien nanties, mais elles seraient aussi culturellement minorisées, donc plus vulnérables lorsqu'il est question de leur santé (Bouchard et collab., 2015; Bouchard et collab., 2009; Forgues, Doucet, Guignard-Noël, 2011).

L'identité territoriale pourrait exercer un effet protecteur sur l'état de santé. En effet, $77 \%$ des aînés francophones des provinces maritimes interrogés dans le cadre de l'ESCC ont affirmé éprouver un fort sentiment d'appartenance à l'égard de leur communauté, contre $81 \%$ chez leurs homologues anglophones et $74 \%$ pour l'ensemble des aînés canadiens. Un tel résultat corrobore les travaux de chercheurs en développement régional, selon lesquels l'appartenance territoriale aurait tendance à se manifester avec une plus forte intensité dans les milieux plus faiblement urbanisés et, de ce fait, constituerait l'un des principaux éléments de caractérisation de la ruralité (Jean, 2012; Carrier, Jean et LeBlanc, 2000).

L'accès aux services de santé poserait aussi problème en milieu minoritaire francophone acadien. À cet égard, l'actualité a permis d'identifier des lacunes en ce qui a trait à l'offre de services offerts en matière de santé dans la région du Restigouche-Ouest au Nouveau-Brunswick. En outre, avec seulement six lits, l'Hôtel-Dieu Saint-Joseph de Saint-Quentin (1532 habitants en 2016) fait piètre figure, si on le compare à son homologue de Perth-Andover (1590 habitants en 2016), qui en possède 22 (Réseau de santé Vitalité, 2020; Réseau de santé Horizon, 2020). Selon le Comité permanent de la santé de Saint-Quentin, ce même centre hospitalier serait aussi désavantagé, comparativement à des établissements de même taille en ce qui a trait aux services d'oncologie, de psychologie, de radiologie ou d'échographie (Infoweekend Edmundston Nord-Ouest, 2018). D'ailleurs, en glanant sur les sites web des hôpitaux de Saint-Quentin et de Perth-Andover, nous avons pu constater que les services offerts dans ce dernier étaient beaucoup plus nombreux par rapport à ceux dispensés dans le premier. Il s'ensuit que les résidents du Restigouche-Ouest doivent se rendre à Campbellton ou à Edmundston, ce qui représente plusieurs dizaines de kilomètres de route ${ }^{3}$, alors que ceux du Haut-de-la-Vallée posséderaient ces mêmes services chez eux. Au stress de la maladie se jouxte trop souvent celui des risques associés à la conduite en période hivernale, et ce, d'autant plus que la route qui relie Saint-Quentin aux deux villes limitrophes n'est pas à quatre voies, en plus d'être particulièrement achalandée par de nombreux poids lourds. En outre, il arrive aussi que certains services soient offerts de manière intermittente à l'hôpital de Campbellton, que ce soit en raison de facteurs structurels (pénurie de personnel) ou conjoncturels (pandémie de la COVID-19) ou des deux (Boisvert, 2020; CIMT-CHAU, 2019).

Par ailleurs, situé à seulement 22 kilomètres de Fredericton, l'hôpital d'Oromocto, qui dessert une population majoritairement anglophone, offre plusieurs services qui ne sont pas offerts à Saint-Quentin. De plus, depuis plusieurs années, les élus de la région du Restigouche-Ouest réclament de meilleurs services ambulanciers en raison des délais d'attente trop élevés (Boisvert, 2020, 2018a, 2017). Dans un article paru dans le quotidien L'Acadie Nouvelle, le Dr Hubert 
Dupuis, président d'Égalité santé en français, a soulevé avec fougue les disparités qui persistent entre les soins de santé pour la population francophone desservie par le Réseau Vitalité et ceux de la population anglophone sous l'égide du Réseau Horizon : «Il y a une réalité pour les anglophones avec une multitude de services, et une pour les francophones qui n'ont que des miettes. C'est extrêmement malheureux. Ça me désole de voir un tel constat après dix années de lutte de notre part» (Boisvert, 2018b). Un tel constat corrobore les résultats d'une étude effectuée par Bouchard et Desmeules (2011). Selon ces auteurs, $45 \%$ de la population interrogée trouve qu'il serait très difficile ou impossible de recevoir des services en français dans les régions du centre et du sud-ouest du Nouveau-Brunswick, par rapport à $11 \%$ pour l'ensemble de la province.

\section{Conclusion}

Au terme de cette réflexion, nous constatons que la population de l'Atlantique, plus spécifiquement celle de l'Acadie des Maritimes, est plus affectée par le vieillissement, comparativement à l'ensemble du Canada. Dans un tel contexte, et compte tenu de sa forte composition rurale et de sa dualité linguistique, la question de la santé des aînés francophones présente un double défi en Acadie des Maritimes, soit l'accès géographique par rapport aux services et, de surcroît, la disponibilité de ceux-ci dans la langue de leur choix. Le rapport minoritaire/majoritaire traduit de toute évidence une inégalité sociale qui, couplée à d'autres déterminants comme le soutien social, l'éducation, l'immigration et la littératie en santé, contribue de facto à la persistance d'inégalités sociales sur le plan de la santé et, de ce fait, à la formation de disparités géographiques.

En ce qui concerne plus spécifiquement la littératie en santé, il appert incontournable de favoriser la prise en compte et le développement des capacités en santé aux aînés francophones vivant en situation linguistique minoritaire. À ce chapitre, ces derniers font face à une double problématique. Premièrement, ils sont bien souvent socialisés dans une langue seconde : l'anglais. Deuxièmement, les personnes âgées, et en particulier celles des provinces maritimes et, de surcroît francophone, sont moins scolarisées et donc moins bien outillés pour comprendre le langage spécialisé de la médecine.
Les effets d'une mauvaise communication en santé, conjugués à ceux d'une minorisation, voire d'une assimilation culturelle toujours bien réelle, amènent à nous interroger sur les pistes de solution à envisager. En premier lieu, nous retenons la nécessité de déployer des actions visant à combler les lacunes en matière de littératie en santé auprès des personnes âgées francophones. Il importe également de mettre en œuvre des démarches visant à bonifier l'offre de services préventifs, éducatifs et de prise en charge en santé auprès des personnes âgées francophones vivant en situation minoritaire. La formation, le financement de "facilitateurs", la création, l'évaluation et l'adaptation d'outils de communication, la disponibilité et l'accessibilité par rapport aux services de proximité, la formation d'intervenants sur les droits linguistiques ainsi que la confection d'un lexique en ligne et sous format papier des termes médicaux apparaissent autant de pistes de solution susceptibles d'améliorer la littératie en santé des personnes âgées en milieu minoritaire francophone.

Les enjeux liés à la santé représentent un défi d'autant plus fondamental dans les localités de petite taille démographique, plus enclines au vieillissement et, de surcroît, en situation de dépeuplement, comme c'est le cas en Acadie des Maritimes. Dans ces milieux, l'embauche d'un agent communautaire pourrait, sans nécessairement faire contrepoids à ces facteurs structurels, contribuer à mieux informer les aînés sur les différents services qui leur sont offerts, en plus de briser leur isolement. Une telle ressource apparaît d'autant plus nécessaire que plusieurs localités rurales, notamment au NouveauBrunswick, ont fait face, au cours des dernières années, à la perte de nombreux services de proximité, dont l'épicerie et la caisse locales. Il importe aussi de réfléchir à de nouvelles modalités concernant l'offre de services de santé, de manière à mieux répondre aux besoins des personnes âgées vivant en contexte minoritaire francophone. À cet égard, la récente pandémie de COVID-19 a permis d'accélérer l'offre de services de santé offerts en ligne ou par téléphone. Une telle pratique est appelée à se déployer dans les prochaines années.

Au final, il s'avère primordial de mieux cerner l'ensemble des déterminants sociaux ainsi que leurs interactions entre les contextes sociolinguistiques, les milieux de vie, la structure spatiale et l'impact des politiques publiques dans un souci d'assurer 
une meilleure équité territoriale. C'est ainsi que l'élaboration d'une stratégie d'aménagement du territoire, adaptée aux diverses réalités des personnes âgées vivant en milieu minoritaire francophone, représente un enjeu crucial en Acadie des Maritimes. Là comme ailleurs, des soins de santé adaptés à la réalité des aînés s'avèrent une condition incontour- nable afin d'améliorer leur qualité de vie, de favoriser un vieillissement actif et en santé, mais aussi et surtout - de promouvoir un développement territorial durable, et ce, non seulement dans le respect et la dignité de la personne humaine, mais aussi dans un double souci de protection de la langue des minorités nationales et d'égalité des chances.

\section{NOTES}

1 Les provinces maritimes renvoient au Nouveau-Brunswick, à l'Île-du-Prince-Édouard et à la Nouvelle-Écosse. Quant à l'Atlantique, elle inclut, outre les trois provinces maritimes, celle de Terre-Neuve-et-Labrador.

2 L'Enquête sur la santé dans les collectivités canadiennes (ESCC) est une enquête transversale découlant d'une initiative de l'Institut canadien d'information sur la santé (ICIS), de Statistique Canada et de Santé Canada. Elle vise à recueillir des renseignements sur l'état de santé, sur l'utilisation des services et sur les déterminants de la santé de la population canadienne. Elle est réalisée depuis 2001 par Statistique Canada auprès d'un échantillon substantiel de répondants et conçue pour fournir des estimations fiables à l'échelle des 133 régions sociosanitaires. Les données pondérées proviennent des cycles combinés de 2003 à 2012. Elles sont basées sur un échantillon de 7640 francophones et de 157312 Canadiens âgés de 65 ans ou plus (excluant le Québec). La combinaison des cycles s'avère nécessaire en raison des faibles échantillons de francophones vivant en situation minoritaire et, de surcroit, d'aînés. Certains résultats présentés ici concernant les francophones ont été publiés dans Bouchard et Desmeules (2017) et Van Kemenade, Bouchard et Bergeron (2015). Ceux portant sur les anglophones sont révélés pour la première fois dans cette contribution. Pour des informations complémentaires concernant l'initiative de l'ICIS, le lecteur est convié à consulter : Institut canadien d'information sur la santé (ICIS), Statistique Canada et Santé Canada. (2020). Enquête sur la santé dans les collectivités canadiennes. Ottawa, $\mathrm{ON}$ : Gouvernement du Canada. Repéré à https://www23.statcan.gc.ca/imdb/p2SV_f.pl?Function=getSurvey\&SDDS=3226\&lang=en\&db=imdb\&adm=8\&dis=2

3 Par exemple, la distance qui sépare Kedgwick et Campbellton est de près de 80 kilomètres. Si les résidents de cette même localité ont à se rendre à Edmundston, c'est plus de 120 kilomètres qu'ils doivent franchir. Dans le cas de Saint-Quentin, la distance qui sépare cette localité de Campbellton et d'Edmundston est respectivement de 96 et de 103 kilomètres.

\section{RÉFÉRENCES}

Audet, M., Paris, M., Garon, S. et Dumas, A. (2017). Saines habitudes de vie, inégalités sociales de santé et vieillissement : réflexions critiques et nouvelles perspectives en développement des communautés. Lien social et Politiques, 78, 254-273. https://doi.org/10.7202/1039348ar

Bélanger, M., Bouchard, L., Gaboury, I., Sonier, B., Gagnon-Arpin, I., Schofield, A. et Bourque, P.-É. (2011). Perceived health status of Francophones and Anglophones in an officially bilingual Canadian province. Canadian Journal of Public Health/Revne canadienne de santé publique, 102(2), 22-126. Repéré à https://www.jstor.org/stable/41995566

Blanchet, B. (2014) Le sens du développement. Communication présentée au Congrès de la Fédération québécoise du développement économique et régional, Québec, Canada.

Blanchet, P. (2005). Minorations, minorisations et minorités : essai de catégorisation d'un processus complexe. Cabiers de sociolinguistique, 10(1), 17-47. https://doi.org/10.3917/csl.0501.0017

Boisvert, J.-F. (2017, 18 août). Accident mortel à Saint-Quentin : une heure avant l'arrivée de l'ambulance. Acadie Nowvelle. Repéré à https://www.acadienouvelle.com/actualites/2017/08/17/accident-mortel-a-saint-quentin-heure-larrivee-de-lambulance

Boisvert, J.-F. (2018a, 13 mars). Il y a un problème avec le service d'Ambulance NB. Acadie Nowvelle, p. 2.

Boisvert, J.-F. (2018b, 20 janvier). Saint-Quentin : « des miettes » pour les francophones. Acadie Nouvelle, p. 6.

Boisvert, J.-F. (2020, 7 avril). Obstétrique et pédiatrie : un arrêt de services qui dérange dans le Restigouche. Acadie Nouvelle, p. 5.

Bouchard, L., Batal, M., Imbeault, P., Sedigh, G., Silva, E. E. et Sucha, E. (2015). Précarité des populations francophones âgées en situation minoritaire linguistique. Minorités linguistiques et société, 6, 66-81. https://doi.org/10.7202/1033190ar

Bouchard, L., Chomienne, M.-H., Benoit, M., Boudreau, F., Lemonde, M. et Dufour, S. (2012). Les Franco-Ontariens âgés souffrant de maladies chroniques se perçoivent-ils bien desservis? Une étude exploratoire de l'impact de la situation linguistique minoritaire. Le médecin de famille canadien, 58(12), 13-25. Repéré à https://www.ncbi.nlm.nih.gov/pmc/articles/PMC3520650 
Bouchard, L. et Desmeules, M. (2011). Minorités de langue officielle du Canada : égales devant la santé? Québec, QC : Presses de l'Université du Québec.

Bouchard, L. et Desmeules, M. (2017). La santé des personnes âgées francophones vivant en situation minoritaire au Canada : enjeux et besoins. Dans M. Drolet, P. Bouchard, et J. Savard (dir.), Accessibilité et offre active : santé et services sociaux en contexte linguistique minoritaire (p. 123-141). Ottawa, ON : Presses de l'Université d'Ottawa.

Bouchard, L., Gaboury, I., Chomienne, M.-H., Gilbert, A. et Dubois, L (2009). La santé en situation linguistique minoritaire. Politiques de santé, 4(4), 36-42. Repéré à https://www.ncbi.nlm.nih.gov/pmc/articles/PMC2700700

Bouchard, L. et Leis, A. (2008). La santé en français. Dans J.-Y. Thériault, A. Gilbert et L. Cardinal (dir.), L'espace francophone en milieu minoritaire an Canada : nowveaux enjeux, nowvelles mobilisations (p. 351-381). Montréal, QC : Fides.

Bowen, S. (2001). Barrières linguistiques dans l'accès aux soins de santé. Ottawa, ON : Santé Canada, Division des systèmes de santé. Repéré à https://www.canada.ca/content/dam/hc-sc/migration/hc-sc/hcs-sss/alt_formats/hpb-dgps/pdf/pubs/2001-langacces/2001-lang-acces-fra.pdf

Bowen, S. (2015). Impact des barrières linguistiques sur la sécurité des patients et la qualité des soins : rapport final. Ottawa, ON : Société santé en français. Repéré à http://francosantesud.ca/wp-content/uploads/SSF-Bowen-S.-\%C3\%89tude-Barri\%C3\%A8reslinguistiques.pdf

Carrier, M., Jean, B. et LeBlanc, P. (2000). Attractivité du territoire, services publics et développement. Dans M. Carrier et S. Côté (dir.), Gouvernance et territoires ruraux : éléments d’un débat sur la responsabilité du développement (p. 85-104). Sainte-Foy, QC : Presses de l’Université du Québec.

Cardinal, L., Langlois, M.-C., Gagné, D. et Tourigny, A. (2008). Perspectives pour un vieillissement en santé : proposition d'un modèle conceptuel. Québec, QC: Agence de la santé et des services sociaux de la Capitale-Nationale.

CIMT-CHAU. (2019, 3 décembre). Les services d'obstétrique suspendus jusqu'au 23 décembre à l'hôpital de Campbellton. Repéré à https:/ / cimtchau.ca/nouvelles/les-services-dobstetrique-suspendus-jusquau-23-decembre-a-lhopital-de-campbellton

Commission mondiale sur l'environnement et le développement. (1988). Notre avenir à tous. Montréal, QC : Éditions du Fleuve et Publications du Québec.

Dugas, C. (1996). L'espace rural canadien. Sainte-Foy, QC : Presses de l’Université du Québec.

El-Batal, K. et Joyal, A. (2015). La Politique nationale de la ruralité québécoise relève-t-elle d’une gouvernance synergique territoriale? Cabiers de géographie, 59(167), 189-207. https://doi.org/10.7202/1036354ar

Epanda, A. (2003). Les milieux ruraux québécois en restructuration: diagnostic, facteurs tangibles et intangibles de dévitalisation rurale et perspectives de développement local approprié (Thèse de doctorat non publiée). Université du Québec à Rimouski, Rimouski, QC. Repéré à https:// constellation.uqac.ca/ 670

Flores, G. (2006). Language barriers to health care in the United States. New England Journal of Medicine, 3(355), 229-231. https://doi.org/10.1056/NEJMp058316

Forgues, É., Doucet, M. et Guignard-Noël, J. (2011). L’accès des ainés francophones aux foyers de soins en milieu minoritaire : un enjeu linguistique en santé et mieux-être. Revue canadienne du vieillissement, 30(4), 603-616. https://doi.org/10.1017/S0714980811000407

Gagnon-Arpin, I., Bouchard. L., Leis, A. et Bélanger, M. (2014). Accès et utilisation des services en langue minoritaire. Dans R. Landry (dir.), La vie dans une langue officielle minoritaire au Canada (p. 193-219). Québec, QC : Presses de l'Université Laval.

Hunt, K. et Macintyre, S. (2000). Genre et inégalités sociales en santé. Dans A. Leclerc, D. Fassier, H. Grandjean, M. Kaminski et T. Lang (dir.), Les inégalités sociales de santé (p. 363-375). Paris, France : La Découverte.

Infoweekend Edmundston Nord-Ouest. (2018, 19 janvier). Soins de santé : le Comité permanent de la santé de Saint-Quentin dénonce les inégalités entre anglophones et francophones. Repéré à https ://www.infoweekend.ca/actualites/actualite/328435/soins-de-sante-le-comitepermanent-de-la-sante-de-saint-quentin-denonce-les-inegalites-entre-anglophones-et-francophones.

Jean, B. (2012). Les territoires ruraux au Québec : vers un modèle de développement territorial durable. Revne d'économie régionale et urbaine, 4(octobre), 649-671. https://doi.org/10.3917/reru.124.0649

Kickbusch, I., Pelikan, J., Apfel, F. et Tsouros, A. D. (2013). Health literacy: The solid facts. Copenhague, Danemark : Organisation mondiale de la santé, Bureau régional européen. 
Klein, J.-L. et Champagne, C. (2011). Initiatives locales et lutte contre la pawureté et l'exclusion. Québec, QC : Presses de l'Université du Québec.

Lagacé, C. (2007). Maintien en emploi, retraite et santé. Québec, QC : Institut national de la santé publique.

Landry, R. (2014). Introduction. Dans R. Landry (dir.), La vie dans une langue officielle au Canada (p. 2). Québec, QC : Presses de l'Université Laval.

Leis, A. et Bouchard, L. (2013). Éditorial : la santé des populations de langue officielle en situation minoritaire. Revne canadienne de santé publique, 104(6), S1-S2. https://www.jstor.org/stable/canajpublheal.104.6.fm

Lord, S. et Piché, D. (2018). Vieillissement et aménagement : perspectives plurielles. Montréal, QC : Presses de l'Université de Montréal.

Meloche, J.-P. (2012). Impacts de la décentralisation fiscale et de la déconcentration sur les disparités régionales : le cas des provinces canadiennes. Revue canadienne des sciences régionales, 35(1), 19-28. Repéré à http://www.cjrs-rcsr.org/V35/1/CJRS-RCSR-35-103Meloche.pdf

Moleux, M., Schaetzel, F. et Scotton, C. (2011). Les inégalités sociales de santé : déterminants sociaux et modèles d'action. Paris, France : Inspection générale des affaires sociales.

Organisation mondiale de la santé (OMS). (2015). Rapport mondial sur le vieillissement et la santé. Résumé. Luxembourg, France : OMS.

Organisation mondiale de la santé (OMS). (2018a). Déterminants sociaux de la santé. Repéré à https://www.who.int/social_ determinants/fr/

Organisation mondiale de la santé (OMS). (2018b). Vieillir en bonne santé: stratégie et plan d'action de l'OMS. Repéré à https://www.who.int/ageing/global-strategy/fr/

Proulx, M.-U. (2019). Splendeurs, misères et ressorts des régions : vers un nouveau gycle de développement régional. Québec, QC : Presses de l’Université du Québec.

Racine, A. (2008). Étude exploratoire sur l'analphabétisme en lien avec la santé et le vieillissement des populations. Québec, QC : Ministère de l'Éducation, du Loisir et du Sport, Direction de l'éducation des adultes et de l'action communautaire.

Rawls, J. (2006). La justice comme équité : une reformulation de Théorie de la justice. Paris, France : La Découverte.

Raymond, É., Gagné, D., Sévigny, A. et Tourigny, A. (2008). La participation sociale des aînés dans une perspective de vieillissement en santé: réflexion critique appuyée sur une analyse documentaire. Québec, QC : Direction de santé publique de l'Agence de la santé et des services sociaux de la Capitale-Nationale.

Réseau de santé Horizon. (2020). Hôpital Hôtel-Dieu Saint-Joseph. Repéré à https:/ / fr.horizonnb.ca/accueil/\%oc3\%a9tablissements-etservices $/ \%$ c3\%a9tablissements $/ \mathrm{h} \%$ c3\%b4tel-dieu-saint-joseph.aspx

Réseau de santé Vitalité. (2020). Hôpital Hôtel-Dieu Saint-Joseph de Saint-Quentin. Repéré à https://www.vitalitenb.ca/fr/points-deservice/hotel-dieu-saint-joseph-de-saint-quentin

Saillant, R. (2016). Deux pays : le Canada à lère du grand déséquilibre démograpbique. Halifax, N.-É. : Nimbus Publishing.

Sarver, J. et Baker, D. W. (2000). Effect of language barriers on follow-up appointments after an emergency department visit. Journal of Internal Medicine, 15(4), 256-264. https://doi.org/10.1111/j.1525-1497.2000.06469.x

Simard, M. (1999). Le Bas-Saint-Laurent : un milieu innovateur. Organisations et Territoires, 8(1-2), 105-112.

Simard, M. (2003). La fragilité de l'espace rural québécois : le cas des petites localités du Bas-Saint-Laurent-Enjeux et perspectives d'avenir (Thèse de doctorat non publiée). Université du Québec à Rimouski, Rimouski, QC. Repéré à https://constellation.uqac.ca/688

Simard, M. (2015). Quelques jalons en vue de l'élaboration d'une politique territoriale du vieillissement au Nouveau-Brunswick. Vie et vieillissement, 12(3), 33-41.

Simard, M. (2018). Le JAL : un demi-siècle de luttes et de débats. Bilan et perspectives nouvelles d'une expérience de développement communautaire en milieu rural fragile. Rimouski, QC : GRIDEQ.

Simard, M. (2020). Le vieillissement de la population en milieu rural québécois. Organisations et Territoires, 29(1), 9-17. 
Simard, M., Dupuis-Blanchard, S., Gould, O., Villalon, L., Éthier, S. et Gibbon, C. (2015). L'influence du contexte sociolinguistique minoritaire sur le maintien à domicile des aînés en milieu rural dévitalisé : le cas d'Acadieville au Nouveau-Brunswick. Revue canadienne du vieillissement, 34(2), 194-206. https://doi.org/10.1017/S0714980815000069

Statistique Canada. (2016a). Espérance de vie, à la naissance et à 65 ans, selon le sexe, moyenne de trois ans, Canada, provinces, territoires, régions sociosanitaires et groupes de régions homologues. Ottawa, ON : Statistique Canada. https://doi.org/10.25318/1310006301-fra

Statistique Canada. (2016b). Recensement de 2016. Ottawa, ON : Statistique Canada. Récupéré à https://www12.statcan.gc.ca/censusrecensement/2016/dp-pd/hlt-fst/lang/Tableau.cfm?lang=E\&T=11\&Geo=00\&SP=1\&view=1\&age=1

Statistique Canada. (2020). Tables de mortalité pour le Canada, les provinces et les territoires. Ottawa, ON : Statistique Canada. Récupéré à https://www150.statcan.gc.ca/n1/pub/84-537-x/84-537-x2019002-fra.htm

Sucha, E., Silva, E., Batista, R. et Bouchard, L. (2015, mai). Mortality in Francophone minorities in Canada: A 16-year follow-up study. Communication présentée à la Conférence annuelle de la Canadian Public Health Association, Toronto, ON.

Tjepkema, M., Wilkins, R. et Long, A. (2012). Mortalité par cause selon le niveau de scolarité au Canada : une étude de suivi de 16 ans. Ottawa, ON : Statistique Canada. Repéré à https://www150.statcan.gc.ca/n1/pub/82-003-x/2012003/article/11700-fra.pdf

Tremblay, D.-G., Klein, J.-L. et Fontan, J.-M. (2009). Initiatives locales et développement socioterritorial. Québec, QC : Presses de l’Université du Québec.

Van Kemenade, S., Bouchard, L. et Bergeron, C. (2015). Enjeux de santé des aînés francophones vivant en situation minoritaire : une analyse différenciée selon les sexes. Reflets, 21(2), 112-130. https://doi.org/10.7202/1035435ar

Wilkins, R., Tjepkema, M., Mustard, C. et Choinnière, R. (2008). Étude canadienne de suivi de la mortalité selon le recensement, 1991 à 2001. Ottawa, ON : Statistique Canada. Repéré à https://www150.statcan.gc.ca/n1/pub/82-003-x/2008003/article/10681-fra.htm

Wilkinson, R. et Pickett, K. (2013). Pourquoi l'égalité est meilleure pour tous. Paris, France : Les petits matins.

Woloshin, S., Schwartz, L. M., Katz, S. J. et Welch, G. H. (1997). Is language a barrier to the use of preventive services? Journal of General Internal Medicine, 12(8), 472-477. https://dx.doi.org/10.1046\%2Fj.1525-1497.1997.00085.x

Yeo, S. (2004). Language barriers and access to care. Annual Review of Nursing Research, 22(1), 59-75. https://doi.org/10.1891/07396686.22 .1 .59

Zorn, N. (2017). Le 1 \% le plus riche : l'exception québécoise. Montréal, QC : Presses de l'Université Laval. 